\title{
Activity and expression of ADP-glucose pyrophosphorylase during rhizome formation in lotus (Nelumbo nucifera Gaertn.)
}

\author{
Libao Cheng ${ }^{1}$, Xian Liu' ${ }^{1}$ Jingjing Yin ${ }^{1}$, Jianqiu Yang ${ }^{1}$, Yan Li ${ }^{1}$, Linchong Hui ${ }^{1}$, Shuyan Li ${ }^{2}$ and Liangjun $\mathrm{Li}^{1 *}$
}

\begin{abstract}
Background: Lotus root is a traditional and popular aquatic vegetable in China. Starch is an important component of the rhizome and directly affects the quality of processed products. ADP -glucose pyrophosphorylase (AGPase) is a rate-limiting enzyme associated with starch biosynthesis in plants. Therefore, in the present study, AGPase activity and NnAGP expression during rhizome development of lotus were analyzed.

Results: Among 15 cultivars analyzed, the contents of amylose and total starch in the rhizome were highest in 'Mei Ren Hong.' 'Su Zhou' and 'Zhen Zhu' showed the lowest amylose, amylopectin and total starch contents. In the rhizome, activity of AGPase was highest at the middle swelling stage of development, and higher activity was observed in the 'Hou ba' leaf and terminational leaf at the same stage. Three AGPase genes, comprising two large subunit genes (NnAGPL1 and NnAGPL2) and one small subunit gene (NnAGPS), were isolated and identified. The deduced amino acid sequences showed $40.5 \%$ similarity among the three genes. Full-length genomic DNA sequences of NnAGPL 1 , NnAGPL2, and NnAGPS were 4841, 11,346 and 4169 bp, respectively. Analysis of the temporal and spatial expression patterns revealed that the transcription levels of NnAGPL1 and NnAGPS were higher in the rhizome, followed by the 'Hou ba' leaf, whereas NnAGPL2 was significantly detected in the 'Hou ba' leaf and terminational leaf. The initial swelling stage of rhizome development was accompanied by the highest accumulation of mRNAs of NnAGPL1, whereas expression of NnAGPL2 was not detected during rhizome development. The transcript level of NnAGPS was highest at the initial swelling stage compared with the other rhizome developmental stages. Transcription of NnAGPL1, NnAGPL2, and NnAGPS was induced within $24 \mathrm{~h}$ after treatment with exogenous sucrose. The mRNA level of NnAGPL1 and NnAGPS was increased by exogenous ABA, whereas transcription of NnAGPL2 was not affected by ABA.
\end{abstract}

Conclusions: The three AGPase genes display marked differences in spatial and temporal expression patterns. Regulation of AGPase in relation to starch synthesis in lotus is indicated to be complex.

Keywords: Lotus root, ADP-glucose pyrophosphorylase, Starch, NnAGP, Expression

\section{Background}

Lotus (Nelumbo nucifera Gaertn.) belongs to the dicot family Nelumbonaceae, which shows many features of monocot plants, and is a popular aquatic herb vegetable (Xue et al. 2012). Lotus originates from India and China, and is widely cultivated in China, Japan, and other Southeast Asian countries for multiple purposes (Sakamoto

\footnotetext{
*Correspondence: ljli@yzu.edu.cn

${ }^{1}$ School of Horticulture and Plant Protection of Yangzhou University, Yangzhou, Jiangsu, People's Republic of China

Full list of author information is available at the end of the article
}

1977). The processed products of the rhizome (often termed lotus root) are in great demand in many countries, including Japan, Korea, the United States and European countries, as a type of off-season vegetable. Lotus root products, including drinks, teas, lotus seeds, fresh, salted, and boiled rhizomes, and lotus root starch, are extremely popular in the daily diet because these products are rich in starch, proteins, vitamins, and minerals (Liu et al. 2010; Slocum and Robinson 1996). In addition, nodus Nelumbinis Rhizomatis, germ, stamens, and lotus root stems are important ingredients in traditional 
medicine (Borgi et al. 2007; Renato et al. 2007; Terashima et al. 2011).

Starch is the most important component present in the rhizome and comprises an average content of $10-20 \%$ of the total fresh weight of the rhizome, but the percentage varies among cultivars. The content and form of starch are critical factors in determining the value and storage of starch-based food and industrial products (Mu-Forster et al. 1996). The structure of the starch granule is complex with two distinct types of polysaccharidic components. The first type is amylose, which consists of many unbranched $\alpha-(1,4)$-linked glucan chains. The enzyme ADP-glucose pyrophosphorylase (AGPase) mediates the biosynthesis of $\alpha(1 \rightarrow 4)$ glucans (Preiss 1988). It catalyzes the synthesis of ADP-glucose from glucose-l-phosphate and ATP in leaves and storage organs (Miiller-Rober et al. 1990). The second type of polysaccharide is amylopectin, which is formed by a large number of straight glucan chains with branching points at $\alpha(1 \rightarrow 6)$ linkages (Le et al. 2010; Vlachos and Karapantsios 2000). The ratio of amylose to amylopectin is the main factor that determines the properties of starch in cultivated species (Fredriksson et al. 1998). In some plants, the starch content in the developing rhizome can be improved by means of variation in the amylose:amylopectin ratio (Policegoudra and Aradhya 2008; Zobel 2006).

The many cultivars of lotus can be classified into two broad categories on the basis of the starch content of the rhizome. The main features of the first category include crispiness owing to low starch content, high water and sugar content, and high crude fiber content. These characteristics often result in precipitation of starch, gelatinization, and low viscosity during product processing, which are responsible for the crispiness, refreshing taste, and good sensory quality of the rhizome. Products of this type, such as salted, frozen, or boiled lotus root, are exported worldwide because of the long shelf life and convenient transportation. The second category of lotus cultivars is characterized by high starch content and low water content. The high content of granular starch gives the rhizome a soft texture, pliability, and increases the viscosity of root-derived products (Wattebled et al. 2002).

In most plants, ADP-glucose pyrophosphorylase is considered to be a key regulatory enzyme in starch synthesis (Laughlin et al. 1998). The enzyme regulates the first step of starch synthesis (Slattery et al. 2000). Starch content is significantly decreased in the endosperm in the shrunken-2 (sh2) mutant of maize, which shows low activity of AGPase, thus indicating the importance of AGPase in starch synthesis (Bhave et al. 1990; Singletary et al. 1997). The Sh2 locus encodes the large subunit of endosperm AGPase (Bhave et al. 1990). The subunit composition of AGPase differs among plant species. The two subunits of AGPase isolated from spinach leaves migrate as 51 and $54 \mathrm{kD}$ proteins, and show major structural differences (Morell et al. 1987). In potato tubers, AGPase is composed of two subunits of similar molecular weight (Sowokinos and Preiss 1982). In maize endosperm, AGPase consists of four subunits (Plaxton and Preiss 1987).

Four types of enzymes, namely AGPase, starch synthase (SS), starch branching enzyme (SBE), and starch debranching enzyme (DBE), are involved in amalgamating the sugar subunits into starch (Hannah and James 2008; Tian et al. 2009). Of these enzymes, AGPase produces ADP glucose and pyrophosphate from glucose1-phosphate and ATP, which is considered to be the first rate-limiting reaction in starch synthesis (Espada 1962; Smidansky et al. 2002). Enhanced expression of the AGPase gene ApL3 can improve starch accumulation in Arabidopsis (Wingler et al. 2000). In addition, an APS gene encoding a small subunit of AGPase, and the genes $A p L 1, A p L 2$, and $A p L 3$ encoding large subunits of AGPase in Arabidopsis have been isolated (Villand et al. 1993). Transcript levels of these four genes are differentially regulated by the stimuli such as sugar and light (Sokolov et al. 1998). It has been documented that AGPase genes is responsive to sugars and ABA in last decades (Fritzius 2001; Rook et al. 2001). The possible reason is that ABA affects the synergistic regulation system involved in starch synthesis (Akihiro et al. 2005). However, the regulation of sugars and ABA on expression of AGPase genes is still unknown. Additional experiments prove that large subunit protein is mainly responsible for starch synthesis (Lin et al. 1988; Wang et al. 1997). Two AGPase genes, encoding small subunits of the protein, have been isolated from Vicia faba. Although expression of both genes showed high correlation with accumulation of starch, the expression levels of the genes were dissimilar at different stages of development (Weber et al. 1995).

Starch is the major storage compound in the lotus rhizome. The starch content directly affects the quality of processed products. Therefore, starch synthesis is a very important process in lotus. Recently, a lotus Wx gene (GBSS), which encodes a granule-bound SS, was isolated and its expression profile was characterized ( $\mathrm{Lu}$ et al. 2012). In the present study, three genes that encode AGPases were isolated using a homolog cloning method. The enzymatic activity and expression of the three genes were studied. The results of these experiments will contribute to an improved understanding of the processes involved in starch synthesis and aid the production of good-quality lotus root products. 


\section{Methods}

\section{Plant material}

Fifteen lotus cultivars that are widely cultivated in China, comprising 'XSBZ,' 'Zao Hua, 'Su Zhou', 'E'lian 6,' 'Xin1', 'JHMRH, 'Xin6,' 'Zao Bai', 'XSHZ, 'JHDZH', 'Zhen Zhu', 'Zhong Hua, 'L0026,' '3735-1' and 'Mei Ren Hong,' were grown at the experimental base of aquatic vegetables in Yangzhou University, Jiangsu, China. Plants were provided with $20-25-\mathrm{cm}$ water depth in spring and average temperatures of $30 / 20^{\circ} \mathrm{C}$ (day/night) for the entire growing season (Cheng et al. 2013).

\section{Measurement of starch content}

Starch content of fifteen lotus was measured according to method described by instruction of 'Guide for modern plant physiology experiments' edited by Shanghai Institute of Plant Physiology, Chinese Academy of Sciences. Three rhizomes per cultivar were collected and oven dried at $150{ }^{\circ} \mathrm{C}$ for $1 \mathrm{~h}$, and then dried to constant weight at $60{ }^{\circ} \mathrm{C}$. The dried material was ground into powder and starch content was determined at $630 \mathrm{~nm}$ with a spectrophotometer (UV2800PC, Hengping, Beijng). Amylose content was measured as described by Niu (1990). $0.1 \mathrm{~g}$ of the sample was placed into a tube and $10 \mathrm{ml}$ of $0.5 \mathrm{~N}$ $\mathrm{KOH}$ was added. the amylose content was measured by comparison against a standard curve.

\section{Estimation of AGPase activity}

Activity of AGPase was measured in accordance with the method of Nakamura et al. (1989). A rhizome sample $(0.6 \mathrm{~g})$ was rinsed and ground into power in $5 \mathrm{ml}$ of $100 \mathrm{mmol} \mathrm{L}^{-1}$ tricine/ $\mathrm{NaOH}$ solution $(\mathrm{pH} 8.0)$ at $0{ }^{\circ} \mathrm{C}$. AGPase activity was measured at $340 \mathrm{~nm}$ wavelength.

For statistical analysis, the data were recorded as the mean \pm SE of three experiments, with about 10 seedlings per experiment. Statistical analyses were performed using the SPSS software ver. 14.0 (SPSS Inc., Chicago, IL, USA).

\section{Isolation of $A G P$ genes}

Total RNA was isolated from ground rhizome samples of the cultivar 'Mei Ren Hong' using RNeasy mini kit (QIAGEN, Hilden, Germany). DNaseI was added to eliminate DNA contamination. The quality of RNA was checked with an ultraviolet spectrophotometer (Eppendorf, Hamburg, Germany). Three gene fragments were cloned using a homolog method, and the PCR products were purified by $1 \%$ agarose gel purification kit, following the manufacturer's instructions (TaKaRa, Tokyo, Japan), and then ligated into the pMD 18-T vector (TaKaRa). Each cloning vector containing the target a gene was transferred into Escherichia coli strain DH5 $\alpha$ cells. The recombinants were spread onto Luria broth medium containing ampicillin, 5-bromo-4-chloro-3-indolyl- $\beta$-D-galactoside, and isopropyl- $\beta$-D-thiogalactopyranoside, and incubated at $37^{\circ} \mathrm{C}$ overnight. White colonies were chosen, placed in liquid LB medium, and incubated with shaking at $37{ }^{\circ} \mathrm{C}$ overnight. After identification of 'positive' clones by PCR amplification, plasmid DNA was extracted using a plasmid Purification Kit (TaKaRa). The plasmids were collected and sequenced by Sangon Biotech (Shanghai, China).

Full-length cDNAs of the three cloned genes were obtained with the random amplification of cDNA ends (RACE) method, using the 3'-Full RACE Core Set Ver.2.0 and $5^{\prime}$-Full RACE Core Set (TaKaRa). Gene-specific outer and inner primers were designed in conserved regions of homolog genes from other plants. Nested PCR was performed using different combinations of the gene-specific primers and the RACE primer. The PCR products were purified, then ligated into the pMD18-T cloning vector (TaKaRa), and subsequently sequenced. For 3'-RACE, the first-strand cDNAs were synthesized using M-MLV reverse transcriptase (TaKaRa) with a 3'-RACE adapter primer. Nested PCR, gel purification, and vector ligation were carried out as described above. Primer synthesis and DNA sequencing were performed by Sangon Biotech. Sequence analysis was undertaken using the DNASTAR software. Knowledge of genomic sequence is indeed important to understand the complete features of a gene. Therefore, for cloning of genomic DNA, the leaf (Mei Ren Hong) was used to amplify full-length DNA by PCR method based on information from the obtained cDNA sequences. All primer sequences are listed in Additional file 1: Table S1.

\section{Gene expression profile analysis}

Temporal and spatial expression of the cloned NnAGP genes were analyzed using quantitative real-time PCR (qPCR) and semi-quantitative reverse transcription-PCR (semi RT-PCR). For analysis of temporal expression, total RNA of 'Mei Ren Hong' was extracted at four stages of rhizome development, namely the stolon period, initial swelling period, middle swelling period, and late swelling period. For spatial expression analysis, total TNA was extracted from five organs (Mei Ren Hong), namely the rhizome, 'Houba' leaf (at this stage, rhizome begins to format), leaf petiole of 'Houba', terminational leaf (the last leaf of lotus) and terminational petiole.

Seedlings at the four-leaf stage were treated with exogenous sucrose $(10 \%)$ and abscisic acid (ABA; $100 \mu \mathrm{M})$ for $24 \mathrm{~h}$. The leaf was used to monitor expression of the three cloned genes. After identification of RNA quality, $2 \mu \mathrm{g}$ of total RNA from each organ was used to synthesize first-strand cDNA using the first cDNA synthesis Kit (Tiangen, China) in accordance with the manufacturer's 
instructions (Tiangen, China). The qPCR reaction was performed with a $\mathrm{Mx}-3000 \mathrm{P}^{\mathrm{TM}}$ qPCR machine (Stratagene, La Jolla, CA, USA). The SYBR ${ }^{\circledR}$ Green Master Mix was used to determine the mRNA level in accordance with the manufacturer's instructions (Tiangen, China). The gene-specific primers and internal standard primers ( $\beta$-actin) are listed in Table 1. The PCR protocol consisted of 35 cycles of $5 \mathrm{~min}$ at $94{ }^{\circ} \mathrm{C}, 30 \mathrm{~s}$ at $94{ }^{\circ} \mathrm{C}, 30 \mathrm{~s}$ at $56{ }^{\circ} \mathrm{C}, 60 \mathrm{~s}$ at $72{ }^{\circ} \mathrm{C}$, and final extension for $10 \mathrm{~min}$ at $72{ }^{\circ} \mathrm{C}$. The PCR products were confirmed by $1 \%$ agarose gel electrophoresis.

\section{Results}

\section{Starch content in the rhizome of lotus cultivars}

Significant differences in amylose, amylopectin and total starch contents of the rhizome were observed among the 15 lotus cultivars. The ranges in amylose, amylopectin and total starch contents observed were $1.86-6.32 \%$, 9.7-14.51 \% and $13.17-18.85 \%$, respectively. The contents of amylose $(11.53 \%)$ and total starch $(18.85 \%)$ for 'Mei Ren Hong' were significantly higher than those of the other cultivars analyzed, and the lowest total starch content was recorded for 'Su Zhou' (13.17 \%). The amylose and amylopectin contents (1.86 and 9.70\%) of 'XSBZ' and 'Zhen Zhu', respectively, were the lowest observed among the cultivars analyzed (Table 1). Therefore, 'Mei Ren Hong' was selected to quantify AGPase activity and for gene expression profile analysis.

ADP-glucose pyrophosphorylase is the first committed enzyme of the starch biosynthesis pathway, and activity of the enzyme affects starch content in plants. In this study, the cultivar 'Mei Ren Hong', which showed the highest starch content in the rhizome, was selected to analyze AGPase activity. Greater activity of AGPase was observed in the 'houba' leaf and termination leaf than in the rhizome, 'houba' leaf petiole and termination leaf petiole. Activity of AGPase was higher at the middle swelling stage than that the other rhizome developmental stages (Fig. 1).

\section{Isolation of $A G P$ genes}

A homolog cloning method combined with the RACE technique was used to isolate full-length cDNAs of three genes from 'Mei Ren Hong'. Comparison of the gene sequences with sequences lodged in the National Center for Biotechnology Information (NCBI) database through tblaxt biological software confirmed that the cDNAs were full-length sequences of AGPase genes. The three genes showed high similarity with AGPL1, AGPL2 and $A G P S$ from other species. Therefore, we designated the genes NnAGPL1, NnAGPL2, and NnAGPS, respectively. The full-length cDNAs of NnAGPL1, NNAGPL2, and NnAGPS were 1587, 1566 and $1945 \mathrm{bp}$, respectively, consisting of a single open reading frame, and encoded a putative polypeptide of 528,521 and 524 amino acids (Additional files 2, 3, 4: Figures S1-S3).

Compared with protein sequences lodged in the NCBI database, NnAGPL1 showed 79, 79, 81, 79 and $79 \%$ sequence similarity with AGPL1 from Prunus persica (EMJ23720), Theobroma cacao (EOY14815), Populus trichocarpa (XP_002300758), Fragaria vesca (XP_004291856) and Vitis vinifera (XP_002281223), respectively. NnAGPL2 showed $100 \%$ similarity with AGPL2 of $V$. vinifera (XP_002281069), Actinidia chinensis

Table 1 Identification of starch content (amylose, amylopectin and total starch content) in different varieties of lotus root including XSBZ, Zao Hua, Su Zhou, E'lian 6, Xin1, JHMRH, Xin6, Zao Bai, XSHZ, JHDZH, Zhen Zhu, Zhong Hua, L0026, 3735-1 and Mei Ren Hong

\begin{tabular}{|c|c|c|c|}
\hline Varieties & Amylose content (\%) & Amylopectin content (\%) & Total starch content (\%) \\
\hline XSBZ & $1.86 \pm 0.015 j$ & $13.42 \pm 0.032 \mathrm{~d}$ & $15.28 \pm 0.047 \mathrm{gh}$ \\
\hline Zao Hua & $2.41 \pm 0.076 i$ & $14.51 \pm 0.020 a$ & $16.92 \pm 0.095 c$ \\
\hline Su Zhou & $2.50 \pm 0.051 i$ & $10.67 \pm 0.020 k$ & $13.17 \pm 0.032 j$ \\
\hline E'lian 6 & $2.52 \pm 0.006 i$ & $12.79 \pm 0.021 f$ & $15.31 \pm 0.017 f g$ \\
\hline Xin1 & $2.68 \pm 0.015 h$ & $13.56 \pm 0.060 c$ & $16.24 \pm 0.045 e$ \\
\hline JHMRH & $2.77 \pm 0.072 h$ & $13.47 \pm 0.032 c$ & $16.24 \pm 0.040 \mathrm{e}$ \\
\hline Xin6 & $2.86 \pm 0.020 \mathrm{~g}$ & $13.88 \pm 0.015 b$ & $16.75 \pm 0.015 d$ \\
\hline Zao Bai & $2.86 \pm 0.115 \mathrm{~g}$ & $14.48 \pm 0.023 a$ & $17.34 \pm 0.096 b$ \\
\hline XSHZ & $3.14 \pm 0.015 f$ & $12.04 \pm 0.021 \mathrm{~g}$ & $15.18 \pm 0.010 h$ \\
\hline $\mathrm{JHDZH}$ & $3.14 \pm 0.015 f$ & $13.12 \pm 0.057 e$ & $16.26 \pm 0.044 \mathrm{e}$ \\
\hline Zhen Zhu & $3.50 \pm 0.025 \mathrm{e}$ & $9.70 \pm 0.095 \mathrm{~m}$ & $13.20 \pm 0.120 j$ \\
\hline Zhong Hua & $3.68 \pm 0.025 d$ & $11.74 \pm 0.010 h$ & $15.42 \pm 0.035 f$ \\
\hline L0026 & $3.95 \pm 0.044 c$ & $10.59 \pm 0.006$ & $14.55 \pm 0.042 i$ \\
\hline $3735-1$ & $5.95 \pm 0.040 b$ & $11.36 \pm 0.010 \mathrm{j}$ & $17.31 \pm 0.050 b$ \\
\hline Mei Rren Hong & $6.32 \pm 0.021 a$ & $11.53 \pm 0.046 i$ & $18.85 \pm 0.067 a$ \\
\hline
\end{tabular}



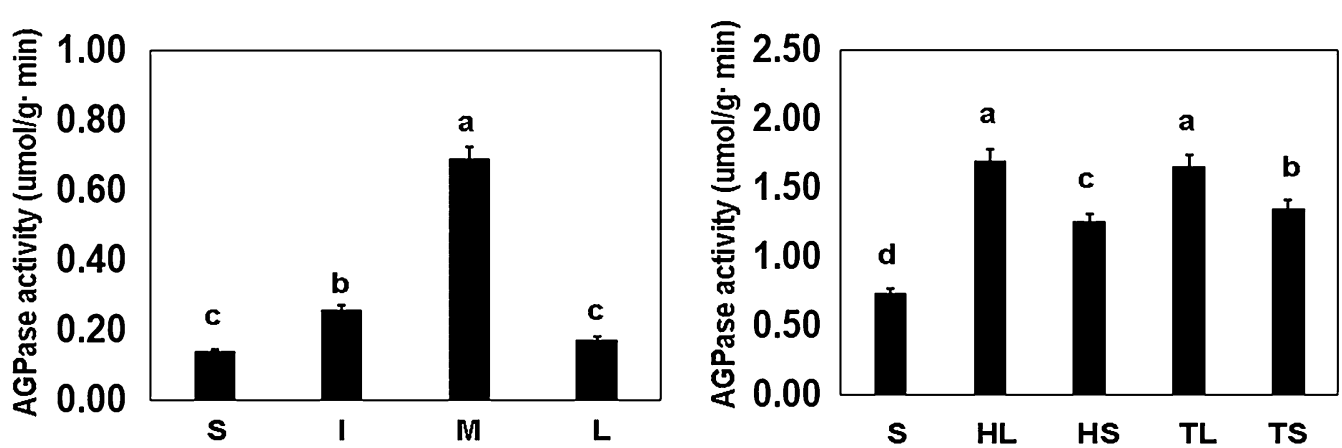

Fig. 1 Activity of AGPase at different developmental stages and organs in lotus root. Activity of AGPase enzyme was determined in crude extracts of lotus organs (rhizome, 'Hou ba' leaf, 'Hou ba' leaf stalk, terminational leaf, terminational leaf stalk) and product organ at different developmental stages (stolon stage, initial swelling stage, middle swelling stage and late swelling stage) as described in "Methods" section

(AFO84093), Ricinus communis (XP_002517196), Theobroma cacao (EOY05585), and Glycine max (XP_003549968), respectively (Fig. 3). NnAGPS showed 99, 91, 90, and $90 \%$ similarity with Nelumbo lutea (AHZ08828.1), Oryza sativa (ACJ86318.1, Ipomoea batatas (AFL55401.1), and Hordeum vulgare (ABX72229.1), respectively (Additional files 5, 6, 7: Figures S4-S6). In addition, $40.5 \%$ similarity in the deduced amino acid sequences of NnAGPL1, NnAGPL2, and NnAGPS was observed (Fig. 2). In addition, phylogenetic analysis of NnAGPL1, NnAGPL2 and NnAGPS with AGPL1, AGPL2 and $A G P S$ of other species showed that NnAGPL1,

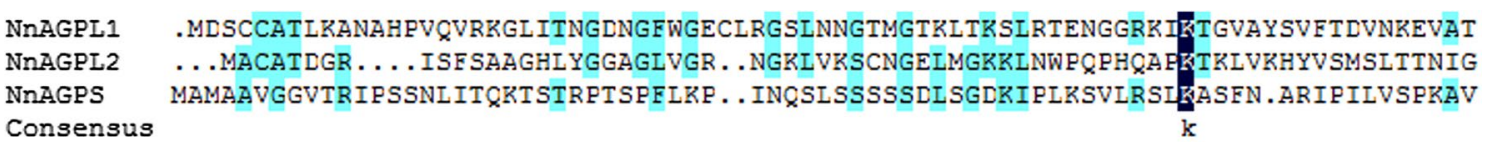

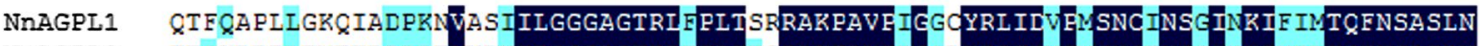
NNAGPL2 IESKLRDLEMEKRDPRTVVAVILGGGAGTRI FPLIRDRAREAVEIGGZYRLIDVENSNCINSGINRIYIITQFNSASLN NRAGPS SDSQGSCTCLDPDASRSVIGIILGGGAGTRIYPLIKKRAKPAVEIGANYRLIDIEVSNCINSNIIIKIYVITQFNSASLN Consensus $\quad \mathrm{v}$ ilgggagtrl plt rakpavp $\mathrm{g}$ yrlid $\mathrm{p}$ snc $\mathrm{ns} i \mathrm{ki}$ tgfnsasln

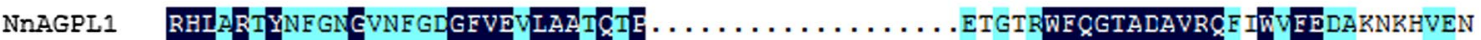
NnAGPL2 RHLZTYNFGN EVNFGLGFVE LAZT NRAGPS RHISEZYASNM GGYKNEGFVEVLAZ DCSEENPNWFQGTADAVRQAQCSPENPNWFCGTADAVRCYIRLFEEHN . . VME Consensus rhl $r y$ g gfve laa q $p$ wfggtadavrq $w$ fe

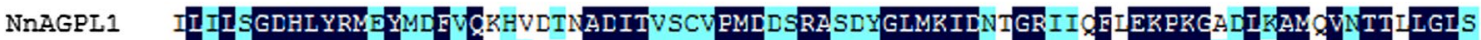

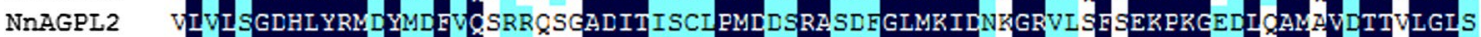

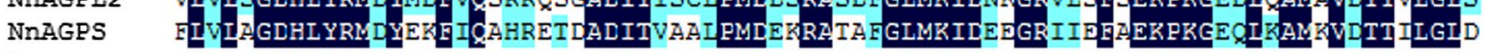
Consensus 1 gdhlyrm $y$ f $q$ adit prd ra glmkid gr $f$ ekpkg $l$ am $v$ tt lgl

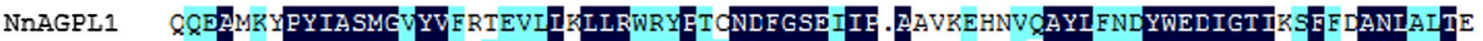

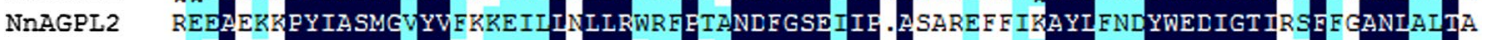

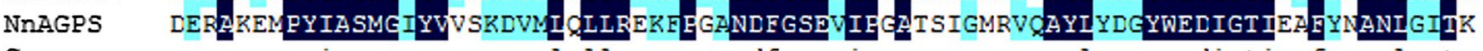

Consensus a pyiasmg yv $l$ llr $p$ ndfgse ip a ayl ywedigti $f$ anl $t$

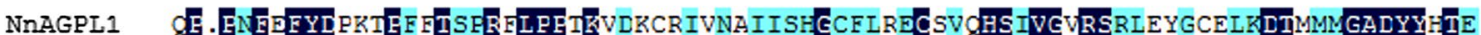

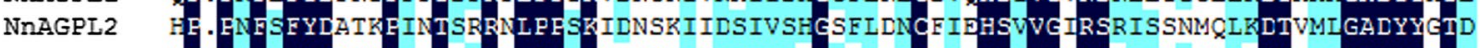
NnAGPS
Consensus $\mathrm{p}$ p f fyd $\mathrm{p} t \mathrm{r}$ lpp $\mathrm{k}$

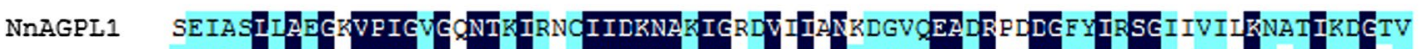
NNAGPL2 AEIASIIZEGRVPIGIGENTKIRECIIDKNA IGKNVVISNSEGIDERDESSDGFYIRSGVTVISKNSIIKLGFV

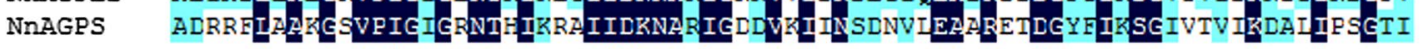
Consensus a $g$ vpig $g$ nt $i$ iidkna ig $v i n$ ea $r$ dg $i s g$ k $i \quad g$

Fig. 2 Alignment of the deduced amino acid of NnAGPL1, NnAGPL2 and NnAGPS 


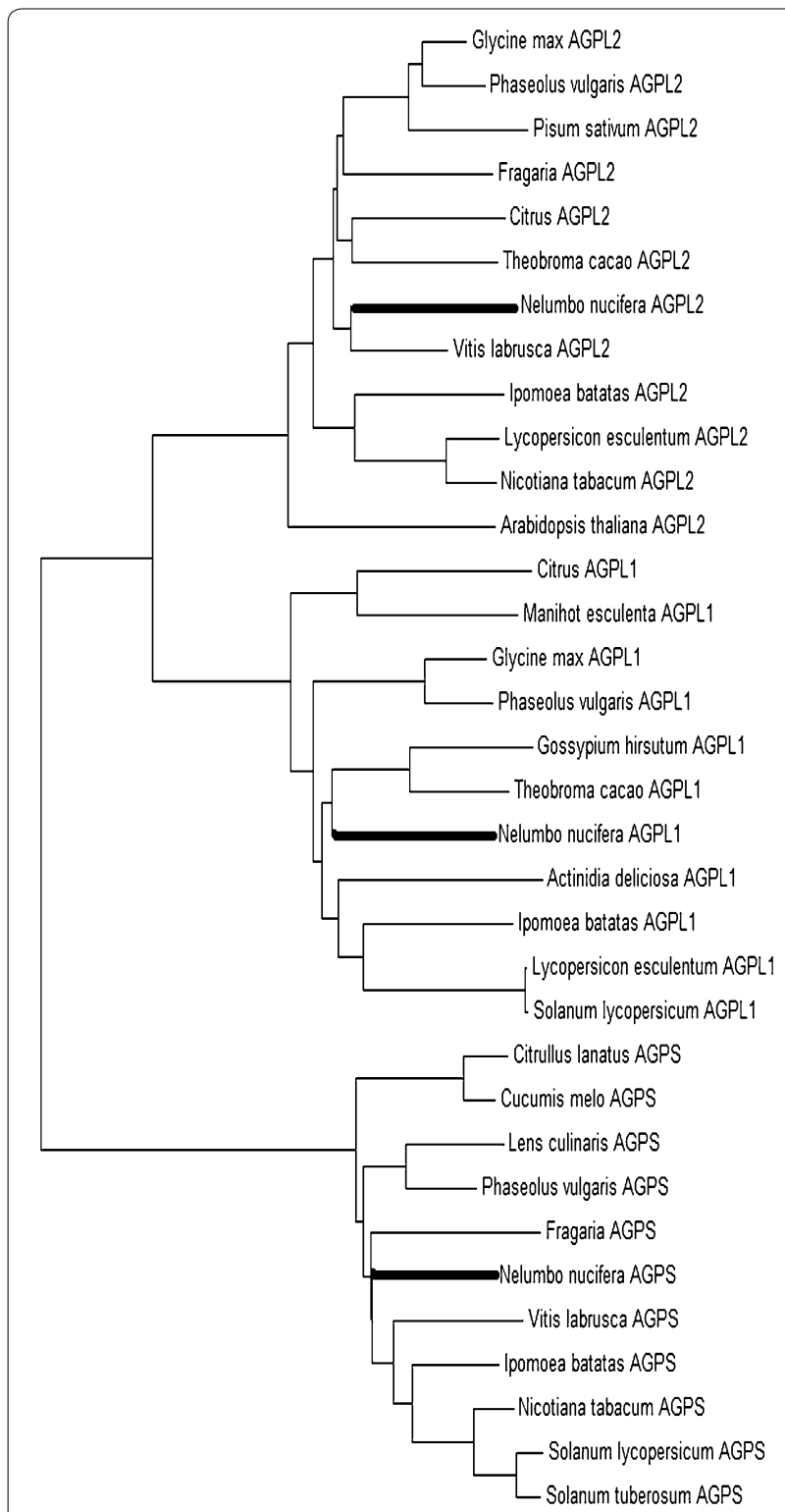

Fig. 3 Analysis of phylogenetic tree of plant NnAGP proteins from different species. The unrooted tree was constructed by using MEGA 4.0 software with a neighbor-joining method. The parameters pairwise deletion and JTT (Jones, Taylor, and Thornton) amino acid substitution model were used. Sequences aligned included AGPL1, AGPL 2, AGPL 3, AGPL 4, and AGPS that were identified or predicted from NCBI database

NnAGPL2 and NnAGPS seemed to have a distant relationship with AGPL1, AGPL2 and AGPS, although they were classified to a group, respectively (Fig. 3).

\section{Genomic DNA sequence and characterization of NnAGPL1,} NNAGPL2, and NnAGPS

Genomic DNA (gDNA) sequences for NnAGPL1, NnAGPL2, and NnAGPS were isolated by PCR method based on information from the obtained cDNA sequences. Information on the primers used is provided in Additional file 1: Table S1. Full length gDNAs of NnAGPL1, NnAGPL2, and NnAGPS were 4841, 11,346 and $4169 \mathrm{bp}$ in length and contained 13, 14 and 8 introns, and 14, 15 and 9 exons, respectively. The seventh, first and first introns in the gDNAs of NnAGPL1, NnAGPL2, and NnAGPS, which were 1049, 1167 and $1175 \mathrm{bp}$, respectively, were the longest introns, whereas the eighth, first and second introns were the shortest. The first, first, and second exon in NnAGPL1, NnAGPL2, and NnAGPS were the longest exons, whereas the sixth, sixth and seventh exons were the shortest, respectively (Table 2).

\section{Expression profiles of NnAGPL1, NnAGPL2, and NnAGPS}

The highest transcript levels for NnAGPL1 were observed in the rhizome and 'Houba' leaf compared with those of the other organs analyzed (Fig. 4A). The mRNA levels detected in the 'Houba' leaf, terminational leaf, and terminational leaf stalk were extremely low. Transcripts of NnAGPL2 were significantly detected in the rhizome (Fig. 4B). The highest levels of NnAGPS mRNAs were detected in 'Houba' leaf stalk and terminational leaf stalk (Fig. 4C).

The expression profiles of NnAGPL1, NnAGPL2, and $N n A G P S$ in the rhizome showed strong temporal differences. The mRNAs of NnAGPL1 were detected at all developmental stages, and the transcript level significantly decreased at the late swelling stage (Fig. 4D). No transcripts of NnAGPL2 were detected in the rhizome at all developmental stages (Fig. 4E). The level of NnAGPS transcripts was observed to be enhanced at the initial swelling, middle swelling, and late swelling stages (Fig. 4F).

Lotus seedlings at the four-leaf stage were treated with sucrose and ABA to monitor changes in NnAGPL1, NnAGPL2, and NnAGPS expression. Transcription of the three genes was significantly enhanced after $18 \mathrm{~h}$ and $24 \mathrm{~h}$ treatment with sucrose (Fig. 4G-I), which indicated that each gene was induced by exogenous sucrose. Transcription of NnAGPL1 and NnAGPS was increased after application of ABA for $12 \mathrm{~h}$, whereas NnAGPL2 was not affected by ABA treatment and showed constitutive expression (Fig. 4J-L).

\section{Discussion}

\section{Starch metabolism during rhizome development}

Many carbohydrate-rich plants, such as rice, maize, lotus, and potato, are loaded with not only ample nutrients, but are also excellent sources of vitamins $\mathrm{A}$ and $\mathrm{C}$ and many other vitamins and minerals (Abbott 1999; Guillon and Champ 2002; De Vries and Toenniessen 2002). 


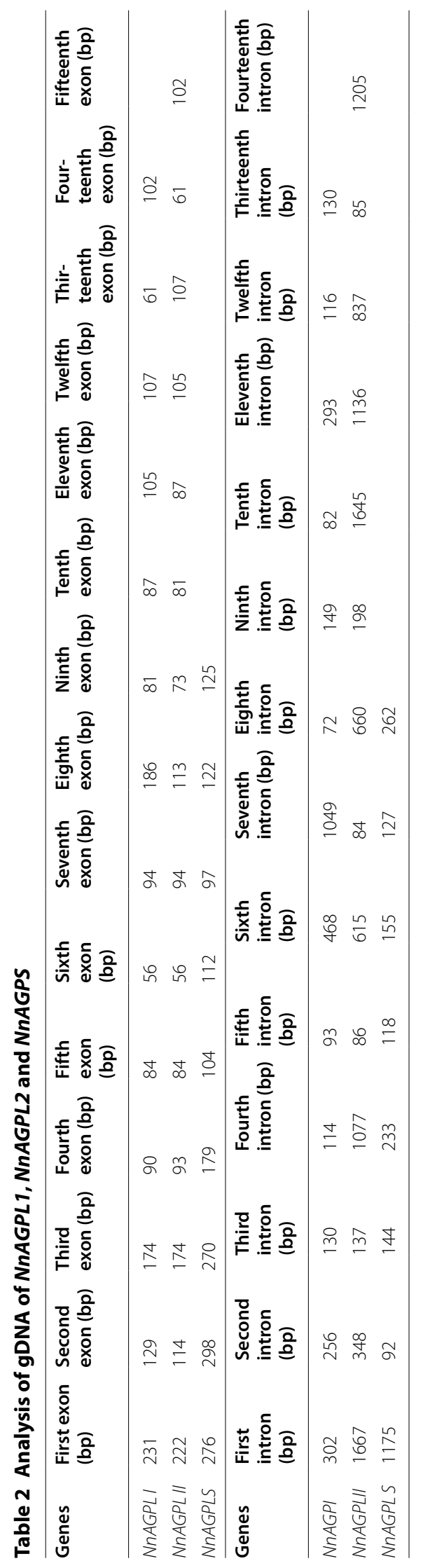




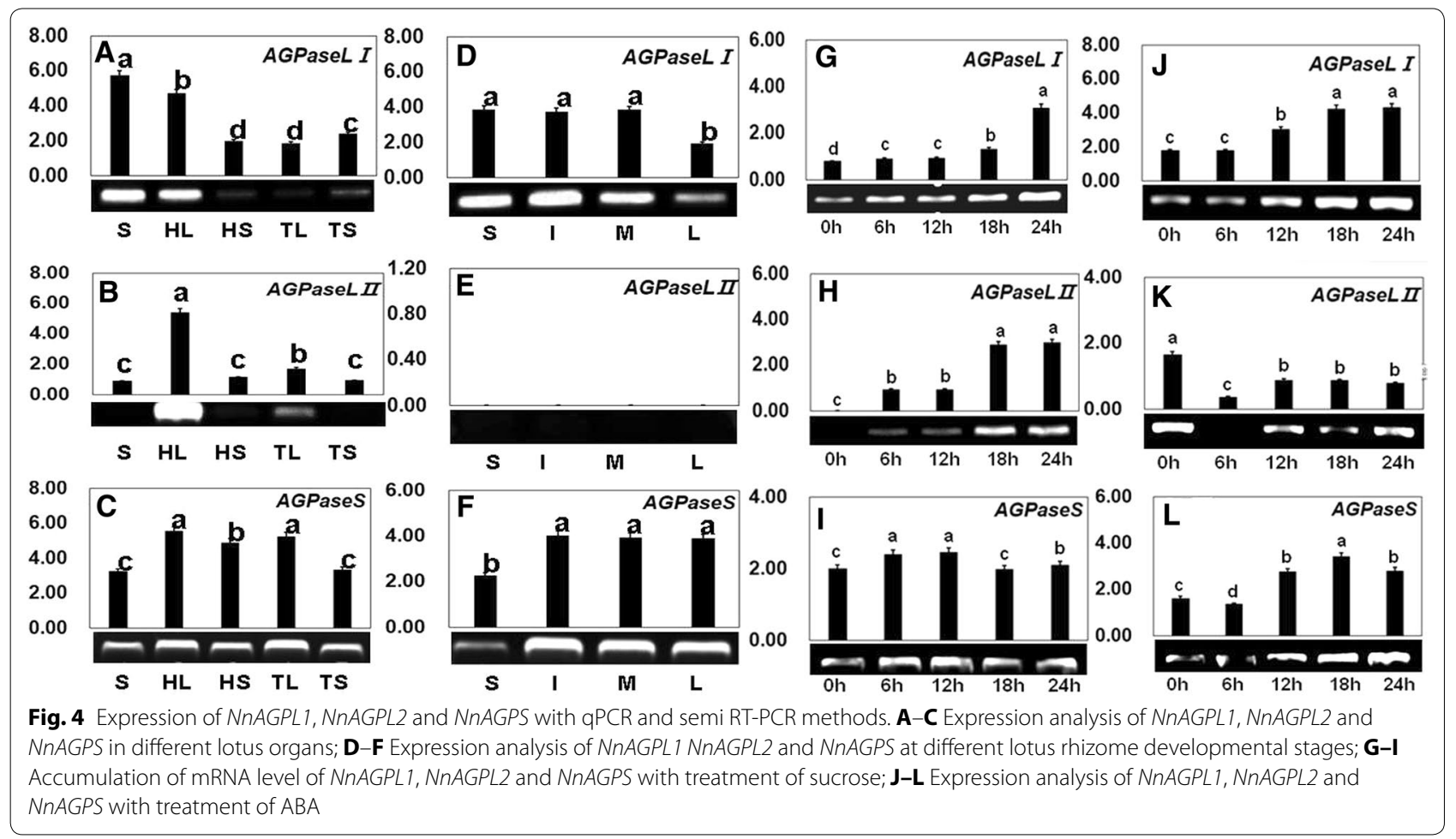

Among carbohydrates, starch provides not only nutrients, but also energy for metabolism through hydrolysis or oxidation reactions during plant growth (Enes et al. 2006; Gao et al. 1998). In China, lotus root is a popular aquatic vegetable. Starch is the most important component present in the rhizome, constituting on average $10-20 \%$ of the total fresh weight. Therefore, the starch content directly affects the quality of lotus root products, and thus is considered the most important qualitydetermining substance. The present results showed that starch content differed significantly among lotus cultivars, which directly determined the end use of the rhizome (Table 1). A high starch content in the rhizome is favored in the Yangtze River region for traditional cooking, thus breeding cultivars with a high starch content in the rhizome is important in many regions of China. It is known that AGPase is an important rate-limiting enzyme in the starch biosynthesis pathway, in both photosynthetic and non-photosynthetic tissues (Pettersson and Ryde-Pettersson 1989; Preiss 1991). In the current study, we observed that AGPase activity showed dissimilarities in different organs and at different developmental stages, which reflected the temporal and spatial regulation of starch synthesis in the rhizome (Fig. 1). Therefore, improvement in AGPase activity in the rhizome by bioengineering approaches is likely to be an efficient means of enhancing the starch content.

\section{Subunits of AGPase}

The molecular mass of AGPase in different organisms ranges from 200 to $400 \mathrm{kD}$ (Weber et al. 1995). Bacterial AGPase consists of four subunits, of which the small unit shows a closer relationship than that of large subunit (Preiss 1991; Weber et al. 1995). In plants, AGPase exists as a heterotetramer, consisting of large and small subunits (Preiss 1991). With respect to the subcellular localization, the plant genome contains four types of AGPase subunits, namely a cytosolic small subunit, plastidial small subunit, cytosolic large subunit, and plastidial large subunit (Burton et al. 2002; Iglesias et al. 1994; Smith-White and Preiss 1992). However, Nakamura and Kawaguchi (1992) observed that AGPase contains six different subunits in the developing endosperm of rice. This phenomenon was confirmed by Akihiro et al. (2005), who identified six genes that encode two small subunits and four large subunits in rice. In lotus, AGPase might consist of two large subunits and one small subunit because we isolated only two NnAGPLs and one NnAGPS from the rhizome on the basis of current genomic information (Additional files 2, 3, 4, 5, 6, 7: Figures S1-S6).

The AGPases localized in the cytosol and plastids are encoded by various genes (Burton et al. 2002; Giroux and Hannah 1994; Johnson et al. 2003). In plants, a mutation in the small subunit of AGPase synthesized in the 
cytoplasm and targeted for plastids does not affect overall activity of AGPase. This finding suggests that small subunits of AGPase localized in the cytosol and plastids are encoded by different genes (Johnson et al. 2003). It also provides a clue to the low sequence homology observed among AGPases (Burton et al. 2002; Johnson et al. 2003). In the present study the two large subunits of NnAGPase showed about $53 \%$ similarity, and only $40.5 \%$ similarity in the deduced amino acid sequences was observed among NnAGPL1, NnAGPL2, and NnAGPS (Fig. 2). These results suggested that the three genes might be synthesized in different organs or serve different functions during starch synthesis.

\section{Structure of gDNA of NnAGPL1, NnAGPL2, and NnAGPS}

Generally, mRNAs that prematurely terminate translation are regulated by nonsense code to maintain a low abundance. This mechanism is present in all eukaryotes to maintain the mRNA level or alter the encoded proteins (Maquat 1996; Ruiz-Echevarria et al. 1996). The two alleles $W x^{a}$ and $W x^{b}$, which are located at the waxy locus, encode starch synthase in cultivated rice. However, their expression profiles are significantly different owing to a different splice site of the first intron, which leads to 10-fold increase in the expression level of $W x^{a}-\mathrm{com}$ pared with that of $W x^{b}$ (Isshiki et al. 1998). The phenomenon of alternative splicing is also common in plants. Different transcripts that encode proteins with functional differences are produced through alternative splicing (Lorkovic et al. 2000). Lotus cultivars are classified into two categories on the basis of the starch content of the rhizome, which determines the end use. In the present study, the cDNAs of NnAGPL1, NnAGPL2, and NnAGPS contained 13, 14 and 8 introns, respectively (Table 2). Interestingly, we determined that the cDNA sequence of NnAGPL1 and 2, NnAGPS in the two categories of lotus are almost identical. This finding suggests that differences in starch content among cultivars might be determined by alternative splicing or different splice sites.

\section{Expression of NnAGP genes in the plant kingdom}

Ample evidence indicates that AGPase, which comprises large and small subunits, plays a critical role in regulating starch synthesis in photosynthetic organs (Preiss and Sivak 1995). Arabidopsis mutants for adg2, which lacks the large subunit of AGPase, show low AGPase activity. This finding indicates that the large protein subunit is essential for AGPase function in starch synthesis (Lin et al. 1988). Although adg2 mutants show some enzyme activity, it also suggests that the small subunit might play an important role (Li and Preiss 1992). The abovementioned reports provide evidence for the importance of both smaller and larger subunits, but that the large subunit makes a greater contribution to AGPase activity (e.g., Johnson et al. 2003; Kang et al. 2010). Kang et al. (2010) observed that two cultivars of winter wheat expressed two identical small subunits, yet the grain starch contents differed. Interestingly, the species with a high starch content in the grain exhibited higher expression levels of the large subunit. These findings suggest that different transcript levels of the large subunit probably contribute to the differences in AGPase activity and rate of starch accumulation (Singh et al. 2001). Over-expression of the large subunit gene can enhance AGPase activity, seed weight, and starch content. Consequently, attention has focused on isolation of the large subunit gene (Li et al. 2010). Analysis of the transcription levels of two large subunit genes was performed with the aim to further explore the mechanisms of starch synthesis in the two types of cultivated lotus that show different starch contents in the rhizome. We observed that NnAGPL1 showed high transcription levels in the rhizome and 'Houba' leaf, whereas transcription of NnAGPL2 was detected only in the 'Houba' leaf (Fig. 4A, B). Expression of NnAGPL1 and NnAGPL2 at specific stages of development indicates that they function in distinct metabolic domains in lotus. In addition, the present results confirmed that transcription of NnAGPL1, NnAGPL2, and NnAGPS showed a high degree of consistency during rhizome development, because rhizome formation starts after emergence of the 'Houba' leaves. In addition, transcription of NnAGPL1 and NnAGPS was closely associated with rhizome formation because these two genes showed high mRNA levels during rhizome formation (Fig. 4D, F).

RNA gel blot analysis of expression of the large subunit gene of sugar beet indicated that the large subunit and small subunit genes were strongly expressed in sink and source leaves (Müller-Röber et al. 1995). A high level of mRNA accumulation for the large subunit gene was observed in the stem of sweet potato, but it was totally absent in the tuber and photosynthetic leaves (Harn et al. 2000; Lee et al. 1999). These results indicate that some large subunit genes may show specific expression patterns in plants. In the present study, we also observed different levels of expression of NnAGP1 and NnAGP2 at different stages of rhizome development (Fig. 4D, E), suggesting that the regulation of AGPase during starch synthesis is complex. Exogenous sugar increases expression of AGPase genes during plant development (Akihiro et al. 2005). Yu et al. (1991) reported that the mRNA level for the $\alpha$-amylase gene of rice is enhanced under sugar starvation, and decreases under sugar abundance, which indicates that plants regulate starch metabolism via the sucrose level (Akihiro et al. 2005). The expression of NnAGPL1, NnAGPL2, and NnAGPS was induced by 
exogenous sucrose (Fig. 4G-I), suggesting that sucrose level might directly regulate starch accumulation through changing the expression of NnAGPL1, NnAGLP2, and NnAGPS in lotus. Expression of some AGPase large subunits is regulated by ABA (Rook et al. 2001). Akihiro et al. (2005) considered that higher plants possess an ABA-regulated system to control starch synthesis, because many starch synthesis-related genes are induced by ABA. The expression of NnAGPL1 was significantly increased after ABA treatment for 18 and $24 \mathrm{~h}$, whereas no change was detected in the transcript levels of NnAGPL2 and NnAGPS (Fig. $4 \mathrm{~J}-\mathrm{L}$ ). This result suggests that the role of NnAGP1 in controlling starch metabolism might be ABA-dependent.

\section{Conclusions}

Based on analysis of starch content and AGPase activity, we isolated three AGPase genes from the lotus rhizome using a homolog cloning method. The genes were designated NnAGPL1, NnAGPL2, and NnAGPS. NnAGPL1 consists of 14 exons and 13 introns, NnAGPL2 contains 15 exons and 14 introns, and NnAGPS consists of nine exons and eight introns. Analysis of the expression of the genes by qPCR and semi RT-PCR shows that the three genes display significant differences in spatial and temporal expression patterns. We conclude that regulation of AGPase in the context of starch synthesis is extremely complex. The present findings when integrated with bioengineering approaches will assist in improving the quality of lotus root products.

\section{Additional files}

Additional file 1: Table S1. Primers for isolation of NnAGPL1, NnAGPL2 and NnAGPS.

Additional file 2: Figure S1. Cloning of full length CDNA and deduced amino acid of NnAGPL1.

Additional file 3: Figure S2. Cloning of full length CDNA and deduced amino acid of NnAGPL2.

Additional file 4: Figure S3. Cloning of full length CDNA and deduced amino acid of NnAGPS.

Additional file 5: Figure S4. Comparison of NnAGPL1 against AGPL1 s of other species.

Additional file 6: Figure S5. Comparison of NnAGPL2 against AGPL2 s of other species.

Additional file 7: Figure S6. Comparison of NnAGPS against AGPS of other species.

\section{Authors' contributions}

$\mathrm{CL}$ was responsible for most data analysis, and wrote the manuscript. LX, YJ, YJ, LY and LS conducted the experimental work. The experiment was designed by $L L$, who critically reviewed the manuscript. All authors read and approved the final manuscript.

\section{Author details}

${ }^{1}$ School of Horticulture and Plant Protection of Yangzhou University, Yangzhou, Jiangsu, People's Republic of China. ${ }^{2}$ College of Guangling, Yangzhou University, Yangzhou, Jiangsu, People's Republic of China.

\section{Acknowledgements}

The authors thank Edanz Group Ltd. for editorial assistance. This work was supported by the Natural Science Foundation of Jiangsu Province, China (BK20151307), Natural Science Fund for Colleges and Universities in Jiangsu Province, China (14KJB210012), the Interdisciplinary Subject Fund of Yangzhou University, China (jcxk2015-15), and the Science and Technology Support Project of Jangsu Province (BE2013388).

\section{Competing interests}

The authors declare that they have no competing interests.

Received: 27 June 2016 Accepted: 20 September 2016

Published online: 30 September 2016

\section{References}

Abbott JA (1999) Quality measurement of fruits and vegetables. Postharvest Biol and and characterization of starch synthase I using mutants in rice. Plant Physiol 140:207-225

Akihiro T, Mizuno K, Fujimura T (2005) Gene Expression of ADP-glucose pyrophosphorylase and starch contents in rice cultured cells are cooperatively regulated by sucrose and ABA. Plant Cell Physiol 46:937-946

Bhave M, Lawrence S, Barton C, Hannah L (1990) Identification and molecular characterization of shrunken -2 cDNA clones of maize. Plant Cell 2:581-588

Borgi W, Ghedira K, Chouchane N (2007) Anti inflammatory and analgesic activities of Zizyphus lutus root barks. Fitoterapia 78:16-19

Burton RA, Johnson PE, Beckles DM, Fincher GB, Jenner HL, Naldrett MJ, Denyer K (2002) Characterization of the genes encoding the cytosolic and plastidial forms of ADP-glucose pyrophosphorylase in wheat endosperm. Plant Physiol 130:1464-1475

Cheng LB, Li SY, Yin JJ, Li L, Chen XH (2013) Genome-wide analysis of differentially expressed genes relevant to rhizome formation in lotus root (Nelumbo nucifera Gaertn). PLoS ONE 8:e67116

De Vries J, Toenniessen G (2002) Securing the harvest: Biotechnology, breeding and seed systems for African crops. The Rockefeller Foundation, New York

Enes P, Panserat S, Kaushik S, Oliva-Teles A (2006) Effect of normal and waxy maize starch on growth, food, utilization and hepatic glucose metabolism in European sea bass (Dicentrarchus labrax) juveniles. Comp Biochem Physiol 143A:89-96

Espada J (1962) Enzymic synthesis of adenosine diphosphate glucose from glucose-1-phosphate and adenosine triphosphate. J Biol Chem 237:3577-3581

Fredriksson H, Silverio J, Andersson R, Eliasson AC, Aman P (1998) The inluence of amylase and amylopectin characteristics on gelatinization and retrogradation properties of different starches. Carbohydr Polym 35:119-134

Fritzius T (2001) Induction of ApL3 expression by trehalose complements the starch-deficient Arabidopsis mutant adg2-1 lacking ApL1, the large subunit of ADP-glucose pyrophosphorylase. Plant Physiol 126:883-889

Gao M, Wanat J, Stinard PS, James MG, Myers AM (1998) Characterization of dull1, a maize gene coding for a novel starch synthase. Plant Cell 10:399-412

Giroux MJ, Hannah LC (1994) ADP-glucose pyrophosphorylase in shrunken-2 and brittle- 2mutants of maize. Mol Gen Genet 243:400-408

Guillon F, Champ MJ (2002) Carbohydrate fractions of legumes; Users in human nutrition and potential for health. Br J Nutr 88:293-306

Hannah LC, James M (2008) The complexities of starch biosynthesis in cereal endosperms. Curr Opin Biotechnol 19:160-165

Harn CH, Bae JM, Lee SS, Min SR, Liu JR (2000) Presence of multiple cDNAs encoding an isoform of ADP-Glucose pyrophosphorylase large subunit from sweet potato and characterization of expression levels. Plant Cell Physiol 41:1235-1242

Iglesias AA, Charng YY, Ball S, Preiss J (1994) Characterization of the kinetic, regulatory, and structural properties of ADP-glucose pyrophosphorylase from Chlamydomonas reinhardtii. Plant Physio 104:1287-1294

Isshiki M, Morino K, Nakajima M, Okagaki R, Wessler SR, Izawa T, Shimamoto K (1998) A naturally occurring functional allele of the rice waxy locus has a GT to TT mutation at the 5 splice of the first intron. Plant J 15:133-138 
Johnson PE, Patron NJ, Bottrill AR, Dinges JR, Fahy BF, Parker ML, Waite DN, Denyer K (2003) A Low-Starch barley mutant, risø16, lacking the cytosolic small subunit of ADP-Glucose pyrophosphorylase, reveals the importance of the cytosolic isoform and the identity of the plastidial small subunit. Plant Physiol 131:684-696

Kang GZ, Wang YH, Liu C, Shen BQ, Zheng BB, Feng W, Guo TC (2010) Difference in AGPase subunits could be associated with starch accumulation in grains between two wheat cultivars. Plant Growth Regul 61:61-66

Laughlin MJ, Chantler SE, Okita TW (1998) N- and C-terminal peptide sequences are essential for enzyme assembly, allosteric, and/or catalytic properties of ADP-glucose pyrophosphorylase. Plant J 14:159-168

Le CD, Bras J, Dufresne A (2010) Starch nanoparticles. Biomacromolecules 11:1139-1163

Lee SS, Bae JM, Oh MS, Liu JR, Harn CH (1999) Isolation and characterization of polymorphic cDNAs partially encoding ADP-glucose pyrophosphorylase (AGPase) large subunit from sweet potato. Mol Cell 10:108-112

Li L, Preiss J (1992) Characterization of ADPglucose pyrophosphorylase from a starch-deficient mutant of Arabidopsis thaliana (L.). Carbohydrate Res 227:227-239

Li N, Zhang SJ, Zhao YJ, Li B, Zhang JR (2010) Over-expression of AGPase genes enhances seed weight and starch content in transgenic maize. Planta 233:241-250

Lin TP, Casper T, Somerville CR, Preiss J (1988) A starch-deficient mutant of Arabidopsis thaliana with low ADPglucose pyrophosphorylase activity lacks one of the two subunits of the enzyme. Plant Physiol 88:1175-1181

Liu J, Zhang M, Wang S (2010) Processing characteristics and flavour of full lotus root powder beverage. J Sci Food Agri 90:2482-2489

Lorkovic ZJ, Kirk DAW, Lambermon MHL, Filipowicz W (2000) Pre-mRNA splicing in higher plants. Trends Plant Sci 5:160-167

Lu Y, Li LJ, Zhou Y, Gao QS, Liang GH, Chen XH, Qi XH (2012) Cloning and characterization of the $W \times$ gene encoding a granule-bound starch synthase in lotus (Nelumbo nucifera Gaertn.). Plant Mol Biol Rep 30:1210-1217

Maquat LE (1996) Defects in RNA splicing and the consequence of shortened translational reading frames. Am J Hum Gen 59:279-286

Miiller-Rober BT, Koflmann J, Curtis Hannah L, Wilimitzer L, Sonnewald U (1990) One of two different ADP-glucose pyrophosphorylase genes from potato responds strongly to elevated levels of sucrose. Mol Gen Genet 224:136-146

Morell MK, Bloom M, Knowles V, Preiss J (1987) Subunit structure of spinach leaf ADPglucose pyrophosphorylase. Plant Physiol 85:182-187

Mu-Forster C, Huang RM, Powers JR, Harriman RW, Knight M, Singletary CW, Keeling PL, Wasserman BP (1996) Physical association of starch biosynthetic enzymes with starch granules of maize endosperm. Plant Physiol 111:821-829

Müller-Röber B, Nast G, Willmitzer L (1995) Isolation and expression analysis of CDNA clones encoding a small and a large subunit of ADP-glucose pyrophosphorylase from sugar beet. Plant Mol Biol 27:191-197

Nakamura Y, Kawaguchi K (1992) Multiple forms of ADP-glucose pyrophosphorylase of rice endosperm. Physiol Plant 84:336-342

Nakamura T, Vrinten P, Hayakawa K, Ikeda J (1998) Characterization of a Granule-Bound Starch Synthase Isoform Found in the Pericarp of Wheat. Plant Physiol 118:451-459

Niu CS (1990) Analysis of crop quality. Agricultural Publishing House of China, Beijing, pp 43-47

Pettersson G, Ryde-Pettersson U (1989) Metabolites controlling the rate of starch synthesis in the chloroplast of C3 plants. Eur J Biochem 179:169-172

Plaxton WC, Preiss J (1987) Purification and properties of nonproteolytic degraded ADP-glucose pyrophosphorylase from maize endosperm. Plant Physiol 83(105-1):12

Policegoudra RS, Aradhya SM (2008) Structure and biochemical properties of starch from an unconventional source- Mango ginger (Curcuma amada Roxb.) rhizome. Food Hydrocolloids 22:513-519

Preiss J (1988) Biosynthesis of starch and its regulation. In: Preiss J (ed) The biochemistry of plant carbohydrate, vol 14. Academic Press, New York, pp 184-249

Preiss J (1991) Biology and molecular biology of starch synthesis and its regulation. Oxford Surv Plant Mol Cell Bio I 7:59-114

Preiss J, Sivak MN (1996) Starch synthesis in sinks and sources. In: Zamski E, Schaffer AA (eds) Photoassimilate Distribution in Plants and Crops: Source-Sink Relationships. Marcel Dekker Inc., New York, pp 63-96
Renato BRAZ, Hechenleitner AAW, Cavalcanti OA (2007) Extraction, structural modification and characterization of lotus roots polysaccharides (Nelumbo nucifera Gaertn). Excipient with potential application in modified drug delivery systems. Lat Am J Pharm 26:706-710

Rook F, Corke F, Card R, Munz G, Smith C, Bevan MW (2001) Impaired sucroseinduction mutants reveal the modulation of sugar-induced starch biosynthetic gene expression by abscisic acid signalling. Plant J 26:421-433

Ruiz-Echevarria MJ, Gonzalez CL, Peltz SW (1996) Making sense of nonsense in yeast. Trends Biochem Sci 21:433-438

Sakamoto Y (1977) Lotus. Hosei University Press, Tokyo [in Japanese]

Singh S, Choi SB, Modi MK, Okita TW (2001) Isolation and characterization of cDNA clones encoding ADP-glucose pyrophosphorylase (AGPase) large and small subunits from chickpea (Cicer arietinum L.). Phytochemistry 59:261-268

Singletary CW, Banisadr W, Keeling P (1997) Influence of gene dosage on carbohydrate synthesis and enzymatic activities in endosperm of starchdeficient mutants of -maize. Plant Physiol 113:293-304

Slattery CJ, Kavakli IH, Okita TW (2000) Engineering starch for increased quantity and quality. Trends Plant Sci 5:291-298

Slocum PD, Robinson P (1996) Water gardening, water lilies and lotuses. Timber Press, Portland

Smidansky ED, Clancy M, Meyer FD, Lanning SP, Blake NK, Talbert LE, Giroux MJ (2002) Enhanced ADP-glucose pyrophosphorylase activity in. PNAS 99:1724-1729

Smith-White BJ, Preiss J (1992) Comparison of proteins of ADP-glucose pyrophosphorylase from diverse sources. J Mol Evol 34:449-464

Sokolov LNV, Dejardin A, Kleczkowski LA (1998) Sugars and light/dark exposure trigger differential regulation of ADP glucose pyrophosphorylase genes in Arabidopsis thaliana (thale cress). Biochem J 336:681-687

Sowokinos JR, Preiss J (1982) Pyrophosphorylases in Solanum tuberosum. III. Purification, physical and catalytic properties of ADP-glucose pyrophosphorylase in potatoes. Plant Physiol 69:1459-1466

Terashima M, Awano K, Honda Y, Yoshino N, Mori T, Fujita H, Ohashi Y, Seguchi O, Kobayashi K, Yamagishi M, Fitzgerald PJ, Yock PG, Maeda K (2011) "Arteries within the artery" after kawasaki disease-a lotus root appearance by intravascular ultrasound. Circulation. doi:10.1161/01. CIR.0000030708.86783.92

Tian ZX, Qian Q, Liu QQ, Yan MX, Liu XF, Yan CG, Liu GF, Gao ZY, Tang SZ, Zeng DL, Wang YH, Yu JM, Gu MH, Li JY (2009) Allelic diversities in rice starch biosynthesis lead to a diverse array of rice eating and cooking qualities. PNAS 106:21760-21765

Villand P, Olsen OA, Kleczkowski LA (1993) Molecular characterization of multiple cDNA clones for ADPglucose pyrophosphorylase from Arabidopsis thaliana. Plant Mol Biol 23:1279-1284

Vlachos NA, Karapantsios TD (2000) Water content measurement of thin sheet starch products using a conductance technique. J Food Eng 46:91-98

Wang SM, Chu B, Lue WL, Yu TS, Eimert K, Chen J (1997) Adg2-1 represents a missense mutation in the ADPG pyrophosphorylase large subunit gene of Arabidopsis thaliana. Plant J 11:1121-1126

Wattebled F, Buleon A, Bouchet B, Jean-Philippe Ral, Lienard L, Delvalle D, Binderup K, Dauvillee D, Ball S, D'Hulst C (2002) Granule-bound starch synthase I-A major enzyme involved in the biogenesis of B-crystallites in starch granules. Eu J Biochem 269:3810-3820

Weber H, Heim U, Borisjuk L, Wobus U (1995) Cell-type specific, coordinate expression of two ADP-glucose pyrophosphorylase genes in relation to starch biosynthesis during seed development of Vicia faba L. Planta 195:352-361

Wingler A, Fritzius T, Wiemken A, Boller T, Aeschbacher RA (2000) Trehalose induces the ADP-Glucose pyrophosphorylase gene, ApL3, and starch synthesis in Arabidopsis. Plant Physiol 124:105-114

Xue JH, Dong WP, Chen T, Zhou SL (2012) Nelumbonaceae: systematic position and species diversification revealed by the complete chloroplast genome. J System Evol. 50:477-487

Yu SM, Kuo YH, Sheu G, Sheu YJ, Liu LF (1991) Metabolic derepression of alphaamylase gene expression in suspension-cultured cells of rice. J Biol Chem 266:21131-21137

Zobel HF (2006) Molecular to granules: a comprehensive starch review. Starch 40:44-50 